\title{
Microcystic adnexal carcinoma in a non-Caucasian patient: A case report and review of the literature
}

\author{
JINGXIN CHEN ${ }^{1,2^{*}}$, SHIPING YANG ${ }^{3 *}$, JIMIN CHEN $^{4}$, TIANAN LIAO ${ }^{1}$, WEI DENG ${ }^{1}$ and WEIZHONG LI $^{2}$ \\ ${ }^{1}$ Department of Oral and Maxillofacial Surgery, Hainan Province People's Hospital, Haikou, Hainan 570311; \\ ${ }^{2}$ Department of Oral and Maxillofacial Surgery, Nanfang Hospital, Southern Medical University, \\ Guangzhou, Guangdong 510515; Departments of ${ }^{3}$ Radiation Oncology and ${ }^{4}$ Pathology, \\ Hainan Province People's Hospital, Haikou, Hainan 570311, P.R. China
}

Received December 4, 2014; Accepted January 11, 2016

DOI: $10.3892 / \mathrm{ol} .2016 .4242$

\begin{abstract}
Microcystic adnexal carcinoma (MAC) is extremely rare among Asians, with the majority of cases presenting in Caucasian individuals. The current study describes the case of a 38-year-old Chinese woman who presented with a 10 year history of a mass in the upper lip. A biopsy resulted in a diagnosis of MAC. The patient underwent complete surgical resection and the tumor was successfully excised. During 6 months of follow-up, there was no evidence of recurrence. To the best of our knowledge, the present case is the first Chinese case to be reported in the English literature, and is presented with the aim of increasing the awareness and aiding in the management of MAC in non-Caucasian populations.
\end{abstract}

\section{Introduction}

Microcystic adnexal carcinoma (MAC) was originally defined in 1982 (1), and is now also termed sclerosing sweat duct carcinoma (2). MAC is a rare, slow-growing, locally aggressive tumor of the eccrine sweat glands, and is characterized by pilar and eccrine differentiation (3). Sweat gland malignancies, combining all types, are particularly rare, accounting for $0.005 \%$ of all malignant epithelial neoplasms (4), with a total of 350 cases currently described in the English literature. It has been reported that the preferential location for the development of this neoplasm is the head and neck region (5).

When referring to the English literature, it is evident that MAC primarily affects the Caucasian population, with only two cases occurring in non-Caucasian patients $(6,7)$. Fang et al (8) described two cases involving Chinese

Correspondence to: Professor Weizhong Li, Department of Oral and Maxillofacial Surgery, Nanfang Hospital, Southern Medical University, 1838 Guangzhou Great Road, Guangzhou, Guangdong 510515, P.R. China

E-mail: liweiz2014@126.com

*Contributed equally

Key words: microcystic adnexal carcinoma, upper lip individuals in the Chinese literature; however, to the best of our knowledge, there are no cases of Chinese patients with MAC currently reported in the English literature. The present study reviewed all patients that were referred to the Hainan Province People's Hospital (Haikou, China) between January 1994 and January 2014, and identified only one patient with a MAC diagnosis. This case is described herein along with a review of the relevant literature.

\section{Case report}

A 38-year-old woman was referred to the Department of Oral and Maxillofacial Surgery at the Hainan Province People's Hospital with a mass on the left upper-lip that had been present for $>10$ years (Fig. 1). During physical examination, it was noted that the tumor was positioned in the left-upper lip and subnasal region. The lesion was a firm, solitary mass, and measured $2 \times 1 \mathrm{~cm}$ in size. No submandibular or cervical lymph nodes were palpable during the physical examination. Abdominal ultrasonography and computed tomography (CT; Brilliance iCT; Philips Medical Systems, Amsterdam, Holland) of the head, neck and thorax demonstrated no evidence of systemic or local metastasis. As presented in Fig. 2, the head and neck CT scans revealed the lesion located in the subcutaneous tissue of the left-upper lip, with a wide base inseparable from overlying skin.

An incisional biopsy was performed with the patient under local anesthesia. The diagnosis of the specimen was confirmed as carcinoma. The patient underwent complete surgical resection of the lesion, with $10 \mathrm{~mm}$ tumor-free margins, which resulted in a subtotal defect of the philtrum and left-upper lip. Reconstruction of the tissue defects was performed immediately, utilizing an adjacent tissue flap of skin and mucous membranes from the cheek (Fig. 3).

Hematoxylin and eosin staining (ZSGB-BIO, Beijing, China) was performed on $4-\mu \mathrm{m}$ thick formalin-fixed and paraffin-embedded resected tumor tissue. Staining was visualized using a BX53 microscope (Olympus Corporation, Tokyo, Japan). Histopathological analysis demonstrated that MAC had infiltrated a number of muscle tissues and peripheral nerve fibers (Fig. 4). Surgical margins were negative. No chemotherapy or radiotherapy regimens were administered, 


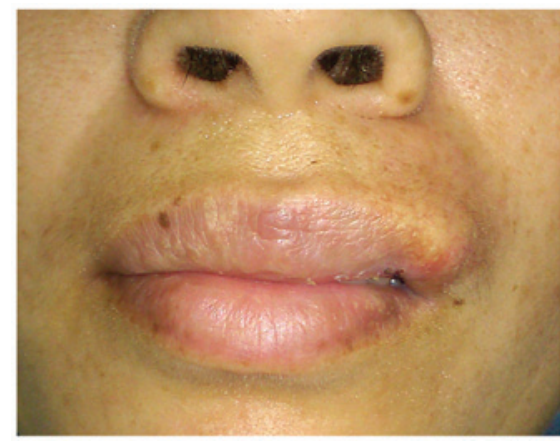

Figure 1. Firm dermal nodule on the left of the upper lip and subnasal region.

A

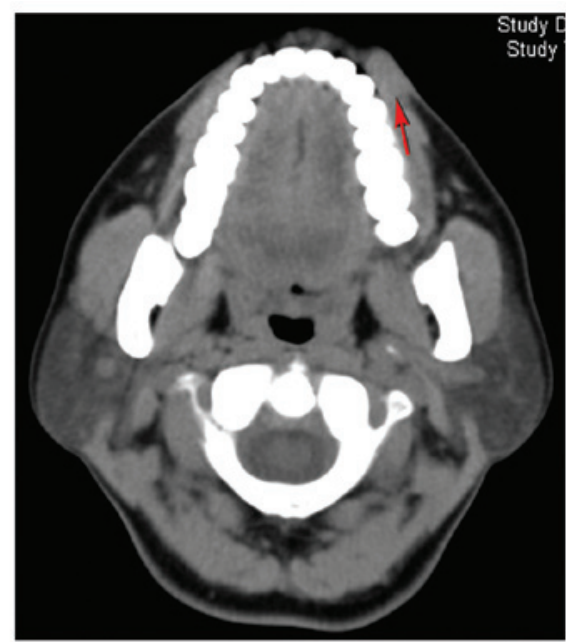

$\mathbf{B}$

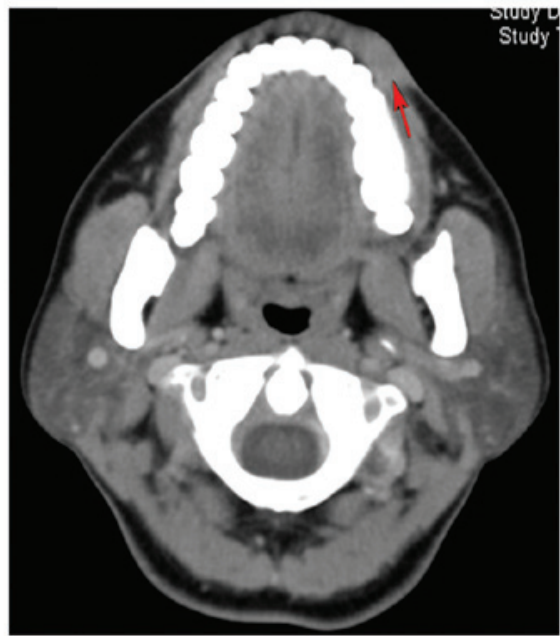

Figure 2. Pre-operative CT of the present case. (A) Contrast axial CT demonstrating the position of the lesion in the subcutaneous tissue of the left upper-lip (arrow), with a wide base that was inseparable from the overlying skin. (B) Axial contrast-enhanced CT demonstrating a slight enhancement of the lesion (arrow). CT, computed tomography.

and no recurrence was observed during 6 months of follow-up. Written informed consent was obtained from the patient for the publication of this study.

\section{Discussion}

The clinical presentation of MAC is characteristically a smooth-surfaced, slow-growing, firm, non-ulcerated,
A

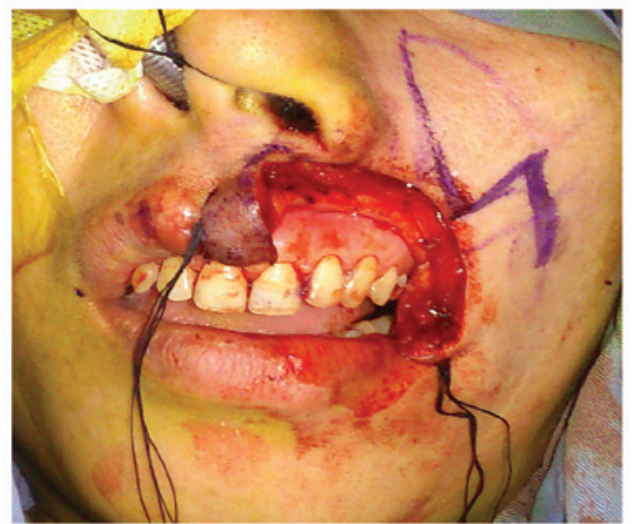

B

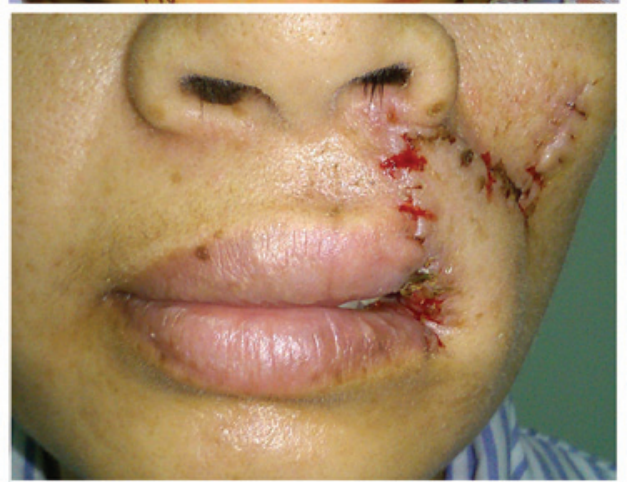

Figure 3. (A) Incision lines for reconstructive surgery marked with blue ink with a rotation flap from the cheek. (B) Post-operative appearance subsequent to reconstruction.

asymptomatic papule, plaque or nodule (9). Nonetheless, a small number of patients are symptomatic at presentation, experiencing burning, paresthesia and numbness as a result of recurrent perineural invasion (9).

It has been reported in the literature that certain MAC cases have presented with tumor histories as long as 17 and 27 years $(3,10)$. Notably, due to the asymptomatic presentation and extremely slow-growth of the tumors, all reported patients have been diagnosed at least 1 year after the initial appearance of the lesions. In the present case, the lesion was present for $>10$ years prior to diagnosis.

Despite the pathogenesis of MAC not yet being fully understood, it is believed to originate from pluripotent keratinocytes, which are capable of differentiation into hair follicles or sweat glands (11). Clinically, the disease presents in the fourth to seventh decades of life, with no evident gender predilection $(11,12)$. In $85 \%$ of cases, the tumors develop in the head and neck area (13), demonstrating a preference for the periorbital skin and the centrofacial region.

MAC is commonly misdiagnosed clinically and histologically, primarily due to its inconspicuous features (14). Rustemeyer et al (15) described a case of the disease that was misdiagnosed as desmoplastic trichoepithelioma for a period of 4 years.

The large majority of reported MAC cases have occurred in Caucasians; however, a small number of reports in the literature have described disease occurrence in non-Caucasians $(6,7)$. To the best of our knowledge, there are currently no reports in the English literature regarding Chinese patients with MAC. However, due to the high prevalence of misdiagnosis and 

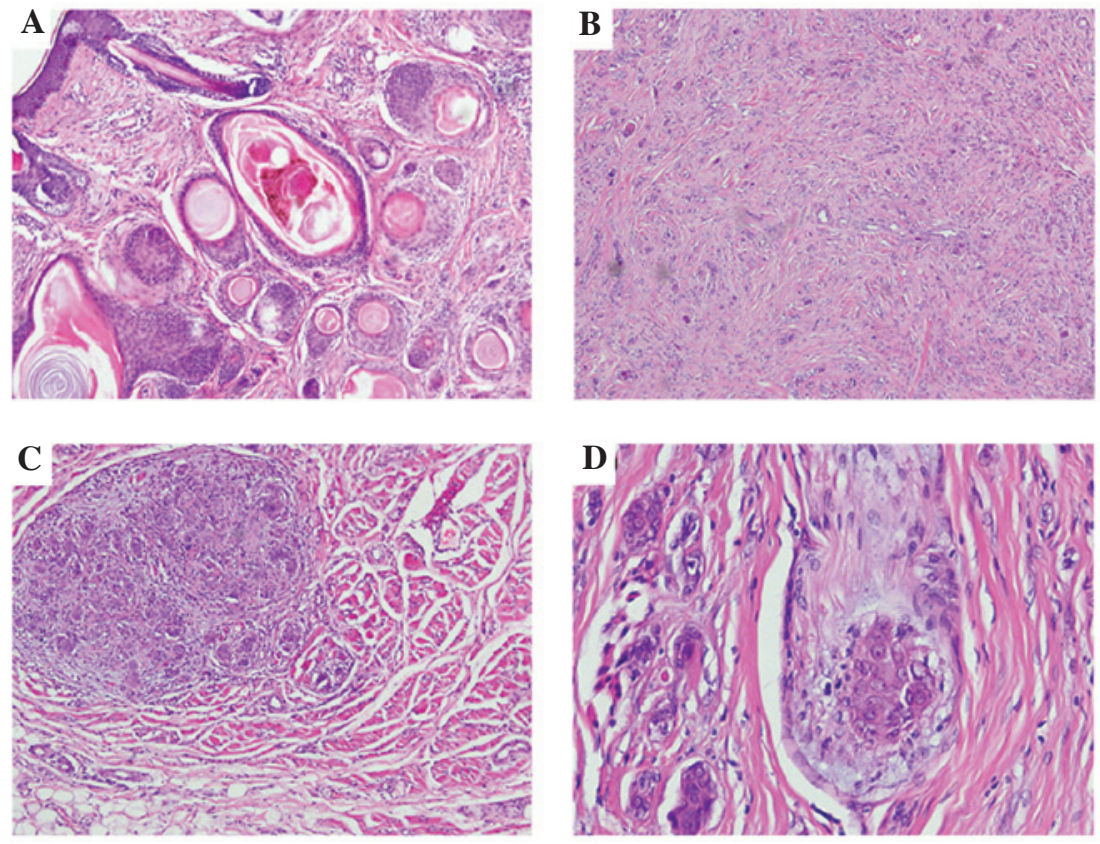

Figure 4. Histological examination of the biopsy tissue. (A) The upper dermis of the tumor exhibiting micronests, cords of cells in dense desmoplastic stroma and keratinous cysts (magnification, x10); (B) Squamoid and glandular clusters are presented in a desmoplastic stroma in thicker sections. Cellular atypia and mitoses are scarce (magnification, x10); (C) MAC-infiltrated muscle tissue (magnification, x10); (D) MAC-infiltrated the peripheral nerve fibers (magnification, $\mathrm{x} 40)$. Staining, hematoxylin and eosin. MAC, microcystic adnexal carcinoma.

possible under-recognition of the disease within this population, a diagnosis of MAC may be significantly overlooked in non-Caucasian individuals as it most commonly occurs in Caucasians. The present case demonstrates the requirement to include MAC in the differential diagnosis in non-Caucasian patients.

The disease presents histologically as a deeply-infiltrative and poorly-circumscribed tumor, consisting of solid aggregates of tumor cells in the mid dermis, superficial keratinous cysts and elongated tubular structures in the deep aspect of the lesion. These cell nests are enveloped by dense, fibrous stroma (16).

MAC is resistant to radiotherapy and chemotherapy, which may be predicted from its slow biological activity (9). The preferred treatment option is complete surgical excision; however, the true tumor-free margins are typically far beyond the intraoperative macroscopically established margins $(17,18)$. Mohs micrographic surgery may be regarded as the gold standard treatment option, with adjuvant radiotherapy and wide local excision offering comparable efficacy (19). When performing adjuvant radiotherapy, a dose of $>50$ Gy should be administered with wide margins $(3-5 \mathrm{~cm})$, owing to the tendency of the lesion for perineural and deep invasion (20). The current patient was not administered any chemotherapy or radiotherapy.

The aggressive and locally invasive nature of the disease results in high recurrence rates, ranging from $15-60 \%$ depending on the treatment modality, with lower rates of recurrence observed following Mohs micrographic surgery (21-24). However, such data may be an overestimate, with misdiagnosis often occurring at the time of excision, and positive margins can occasionally be misread.

It has been reported that tumor recurrence may occur up to 30 years after the initial surgical excision (12), highlighting the importance of regular, long-term follow-up.
Regional and distant metastasis have been described in the literature; however, such cases are particularly rare, with only five cases of local recurrence and three cases of distant metastasis reported $(25,26)$. According to the relevant literature, only one mortality has occurred as a result of the disease (27).

MAC is extremely rare among non-Caucasians, with the majority of cases presenting in Caucasian individuals. The present case describes the occurrence of MAC of the lip in a Chinese woman. To the best of our knowledge, the current case is the first of its kind to be reported in the English literature. The case highlights the importance of the inclusion of MAC within the differential diagnosis in non-Caucasian individuals, and additionally aims to increase the awareness and aid the management of MAC in non-Caucasian populations.

\section{References}

1. Goldstein DJ, Barr RJ and Santa Cruz DJ: Microcystic adnexal carcinoma: A distinct clinicopathologic entity. Cancer 50: 566-572, 1982

2. Cooper PH, Mills SE, Leonard DD, et al: Sclerosing sweat duct (syringomatous) carcinoma. Am J Surg Pathol 9: 422-433, 1985

3. Nickoloff BJ, Fleischmann HE, Carmel J, Wood CC and Roth RJ: Microcystic adnexal carcinoma: Immunohistologic observations suggesting dual (pilar and eccrine) differentiation. Arch Dermatol 122: 290-294, 1986.

4. Wick MR, Goellner JR, Wolfe JT III and Su WP: Adnexal carcinomas of the skin. I. Eccrine carcinomas. Cancer 56: 1147-1162, 1985.

5. Yu JB, Blitzblau RC, Patel SC, Decker RH and Wilson LD: Surveillance, Epidemiology, and End Results (SEER) database analysis of microcystic adnexal carcinoma (sclerosing sweat duct carcinoma) of the skin. Am J Clin Oncol 33: 125-127, 2010.

6. Gardner ES and Goldberg LH: Neglected microcystic adnexal carcinoma: The second reported case in a black patient. Dermatol Surg 27: 678-680, 2001.

7. Park JY and Parry EL: Microcystic adnexal carcinoma. First reported case in a black patient. Dermatol Surg 24: 905-907, 1998. 
8. Fang W, Chen D, Shang JF, Wang F and Xiao L: Microcystic adnexal carcinoma: Report of two cases. Zhonghua Bing Li Xue Za Zhi 38: 59-60, 2009 (In Chinese).

9. Wetter R and Goldstein GD: Microcystic adnexal carcinoma: A diagnostic and therapeutic challenge. Dermatol Ther 21 : 452-458, 2008.

10. Miyamoto T, Kambe N, Nishiura S, Mihara M and Shimao S: Microcystic adnexal carcinoma. Electron microscopic and immunohistochemical study. Dermatologica 180: 40-43, 1990.

11. LeBoit PE and Sexton M: Microcystic adnexal carcinoma of the skin. A reappraisal of the differentiation and differential diagnosis of an underrecognized neoplasm. J Am Acad Dermatol 29: 609-618, 1993.

12. Burns MK, Chen SP and Goldberg LH: Microcystic adnexal carcinoma: Ten cases treated by Mohs micrographic surgery. J Dermatol Surg Oncol 20: 429-433, 1994.

13. Thomas CJ, Wood GC and Marks VJ: Mohs micrographic surgery in the treatment of rare aggressive cutaneous tumors: The Geisinger experience. Dermatol Surg 33: 333-339, 2007.

14. Wu-Chen WY, Weng CY, Rajan KD, Eberhart C and Miller NR: Unusual presentation of primary orbital microcystic adnexal carcinoma. J Neuroophthalmol 31: 147-150, 2011.

15. Rustemeyer J, Zwerger S, Pörksen M and Junker K: Microcystic adnexal carcinoma of the upper lip misdiagnosed benign desmoplastic trichoepithelioma. Oral Maxillofac Surg 17: 141-144, 2013

16. Klein W, Chan E and Seykora JT: Tumors of the epidermal appendages. In: Lever's Histopathology of the Skin. Elder DE (ed) 9th edition. Lippincott Williams \& Wilkins, Edinburgh, pp897-898, 2005.

17. Lober CW and Larbig GG: Microcystic adnexal carcinoma (sclerosing sweat duct carcinoma). South Med J 87: 259-262, 1994.

18. Mayer MH, Winton GB, Smith AC, Lupton GP, Parry EL and Shagets FW: Microcystic adnexal carcinoma (sclerosing sweat duct carcinoma). Plast Reconstr Surg 84: 970-975, 1989.
19. Chaudhari SP, Mortazie MB, Blattner CM, Garelik J, Wolff M, Daulat J and Chaudhari PJ: Treatments for microcystic adnexal carcinoma - A review. J Dermatolog Treat 11: 1-7, 2015 (Epub ahead of print).

20. Baxi S, Deb S, Weedon D, Baumann K and Poulsen M: Microcystic adnexal carcinoma of the skin: The role of adjuvant radiotherapy. J Med Imaging Radiat Oncol 54: 477-482, 2010.

21. Chiller K, Passaro D, Scheuller M, Singer M, McCalmont T and Grekin RC: Microcystic adnexal carcinoma: Forty-eight cases, their treatment and their outcome. Arch Dermatol 136: 1355-1359, 2000.

22. Nelson PS, Bourgeois KM, Nicotri T Jr, Chiu ES and Poole JC: Sclerosing sweat duct carcinoma in a 6-year-old African American child. Pediatr Dermatol 25: 38-42, 2008.

23. Cooper PH: Sclerosing carcinomas of sweat ducts (microcystic adnexal carcinoma). Arch Dermatol 122: 261-264, 1986

24. Leibovitch I, Huilgol SC, Selva D, Lun K, Richards S and Paver R: Microcystic adnexal carcinoma: Treatment with Mohs micrographic surgery. J Am Acad Dermatol 52: 295-300, 2005.

25. Gabillot-Carré M, Weill F, Mamelle G, Kolb F, Boitier F, Petrow P, Ortoli JC, Margulis A, Souteyrand P, Mercier S, et al: Microcystic adnexal carcinoma: Report of seven cases including one with lung metastasis. Dermatology 212: 221-228, 2006

26. Ohta M, Hiramoto $M$ and Ohtsuka H: Metastatic microcystic adnexal carcinoma: An autopsy case. Dermatol Surg 30: 957-960, 2004.

27. Peterson CM, Ratz JL and Sangueza OP: Microcystic adnexal carcinoma: First reported case in an African American man. J Am Acad Dermatol 45: 283-285, 2001 